\title{
PLANTA DIDÁTICA SMAR PD3: MODELAGEM, SIMULAÇÃO E AJUSTE DOS PARÂMETROS DO CONTROLADOR DE TEMPERATURA DO TANQUE DE MISTURA
}

\section{DIDACTIC PLANT SMAR PD3: MODELING, SIMULATION AND ADJUSTMENT OF THE TEMPERATURE CONTROLLER PARAMETERS OF THE MIXING TANK}

\author{
N. C. GOMES ${ }^{1}$, J. V. NICACIO ${ }^{2}$ e A. G. TÔRRES ${ }^{3}$ \\ ${ }^{1}$ Universidade Federal de Viçosa, Departamento de Química, Brasil \\ ${ }^{2}$ Universidade Federal de Viçosa, Departamento de Engenharia Agrícola, Brasil \\ ${ }^{3}$ Universidade Federal de Viçosa, Departamento de Engenharia Elétrica, Brasil \\ E-mail: nubiacostagomes@gmail.com
}

article info

Article history:

Received 2017-07-12

Accepted 2017-10-06

Available online 2017-11-20
PALAVRAS-CHAVE: Processos Químicos; Planta SMAR PD3; Modelagem; Simulação; Controle Temperatura.

KEYWORDS: Chemical Processes; SMAR PD3 Plant; Modeling; Simulation; Temperature Control.

RESUMO: $O$ trabalho teve por objetivo implementar um controle de temperatura no tanque de mistura da planta SMAR PD3. As modelagens teórica e experimental do tanque foram realizadas, assim como a sintonia do controlador PI. Para a modelagem experimental, os parâmetros da função de transferência do processo de mistura no tanque foram determinados através da análise das curvas de variação da temperatura no tanque em resposta a degraus aplicados na vazão fria nos valores de 100, 200 e 400 L.h. ${ }^{-1}$. A sintonia do controlador PI foi realizada para o volume intermediário do tanque e, em seguida, o controle foi validado experimentalmente na planta. Três sintonias foram determinadas com respostas satisfatórias, especialmente a sintonia $2\left(K_{C}=-2250\right.$ e $\left.\tau_{I}=88\right)$, que atingiu o set-point desejado em menos de 4 minutos, para processos onde uma resposta mais rápida é desejada e a sintonia $3\left(K_{C}=-1875\right.$ e $\left.\tau_{I}=88\right)$ para processos onde oscilações não são desejadas ou não são permitidas.

\begin{abstract}
The objective of the present work was to implement a temperature control in the mixing tank of the SMAR PD3 plant. The theoretical and experimental models of the tank were performed, as well as the tuning of the PI controller. For the experimental modeling, the parameters of the transfer function of the mixing process in the tank were obtained by analyzing the temperature variation curves in the tank in response to steps applied in the cold flow in the values of 100, 200 and $400 \mathrm{~L} . \mathrm{h}^{-1}$. The tuning of the PI controller was performed for the medium volume of the tank and then the control was validated experimentally in the plant. Three tunings were determined with satisfactory responses, especially tuning $2\left(K_{C}=-2250\right.$ e $\left.\tau_{I}=88\right)$, which reached the desired set-point in less than 4 minutes, for processes where a faster response is desired and tuning $3\left(K_{C}=-\right.$ 1875 e $\tau_{I}=88$ ) for processes where oscillations are not desired or not allowed.
\end{abstract}




\section{INTRODUÇÃO}

A planta química é um conjunto de unidades processadoras (reatores, trocadores de calor, bombas, evaporadores, tanques), integradas umas com as outras de maneira sistêmica e racional, que tem por objetivo global a conversão de matérias-primas em produtos desejados, por intermédio do uso mais econômico de fontes de energia disponíveis.

Nos últimos anos, o desempenho requerido nas plantas de processos tem se tornado cada vez mais difícil de satisfazer. A alta competitividade do mercado, as regulamentações ambientais e de segurança e as rápidas mudanças nas condições econômicas têm sido fatores determinantes no aumento das especificações de qualidade. Com uma tendência para processos cada vez mais complexos e altamente integrados é natural que o controle de processos se tornasse cada vez mais importante. Seria impossível operar plantas modernas de forma segura e rentável e ainda satisfazer a qualidade do produto e requisitos ambientais sem sistemas de controle de processos automatizados (SEBORG et al., 2003).

De acordo com Seborg et al. (2003), o projeto e a implementação de um sistema de controle envolvem vários passos. O primeiro deles é a definição dos objetivos do controle. Esta definição é feita com base nos objetivos de operação para a planta e nas restrições do processo. Após isso, um modelo dinâmico é desenvolvido para o processo, que pode ser teórico, experimental ou híbrido. Com dados experimentais da planta disponíveis, o modelo dinâmico é validado e um modelo de precisão é identificado. Essa informação é usada para o sistema de controle e sintonia do controlador.

Em seguida a estratégia de controle é planejada, etapa que envolve o entendimento do processo, experiência e preferências no projeto. Simulações do processo em computador geralmente são usadas para visualizar alternativas de estratégias de controle e para fornecer iniciais estimativas para as configurações mais adequadas do controlador. Como ponto de partida, o sistema de controle é implementado na planta usando as estimativas preliminares do projeto. Em seguida, procedimentos de tentativa e erro são frequentemente usados na sintonização final do controlador.

As plantas didáticas têm um papel muito importante no estudo de controle de processos de plantas industriais. A planta didática SMAR PD3, por exemplo, tem por objetivo demonstrar didaticamente a operação das diversas malhas de controle utilizando equipamentos e ferramentas de configuração similares àqueles de aplicação de controle industrial. Seu arranjo compacto permite realizar estudos, treinamentos e experimentos com diversos tipos de controle nas principais variáveis de processo como temperatura, pressão, vazão e nível, possibilitando aos seus usuários experiências similares às que irão encontrar no mercado de trabalho.

Morais et al. (2017) e Domingos et al. (2017) apresentam o desenvolvimento de um controlador de temperatura para o tanque de aquecimento da mesma planta didática SMAR PD3 utilizada neste trabalho. A modelagem do tanque de aquecimento foi feita através do estudo da resposta transiente ao degrau de potência para vazões constantes de 200, 400, 600 e $1000 \mathrm{~L} / \mathrm{h}$. Após validação do modelo, a sintonia do controlador PI da planta foi realizada através do Método do Lugar das Raízes, o que permitiu o controle do processo de 
aquecimento no tanque para diversas condições.

Gomes (2013) também compara a implementação de controladores PID e Fuzzy na planta piloto SMAR PD3 instalada no Instituto Federal Fluminense. O controle de temperatura do tanque de aquecimento da planta foi testado através dos dois controladores e a atuação dos mesmos foi comparada. Os resultados obtidos permitiram concluir que o controlador PID apresenta uma ação corretiva mais eficiente, já que apresentou respostas mais próximas do valor desejado e em menor tempo de resposta em relação ao controlador Fussy.

Dessa forma, o objetivo deste trabalho foi realizar a modelagem teórica e experimental do tanque de mistura da planta didática SMAR PD3, onde ocorre o processo de mistura de duas vazões com temperaturas diferentes, a fim de identificar um modelo de precisão que caracterize o processo, além de implementar um controle de temperatura no respectivo tanque através da sintonia do controlador PI da planta pelo método do Lugar das Raízes.

\section{MATERIAL E MÉTODOS}

\subsection{Funcionamento Planta Didática SMAR PD3}

Os experimentos foram realizados na planta didática SMAR PD3, ilustrada na Figura 1, localizada no Laboratório de Pesquisa de Processos Industriais e Padrões de Movimentos e Acústica do Departamento de Engenharia Elétrica da Universidade Federal de Viçosa.

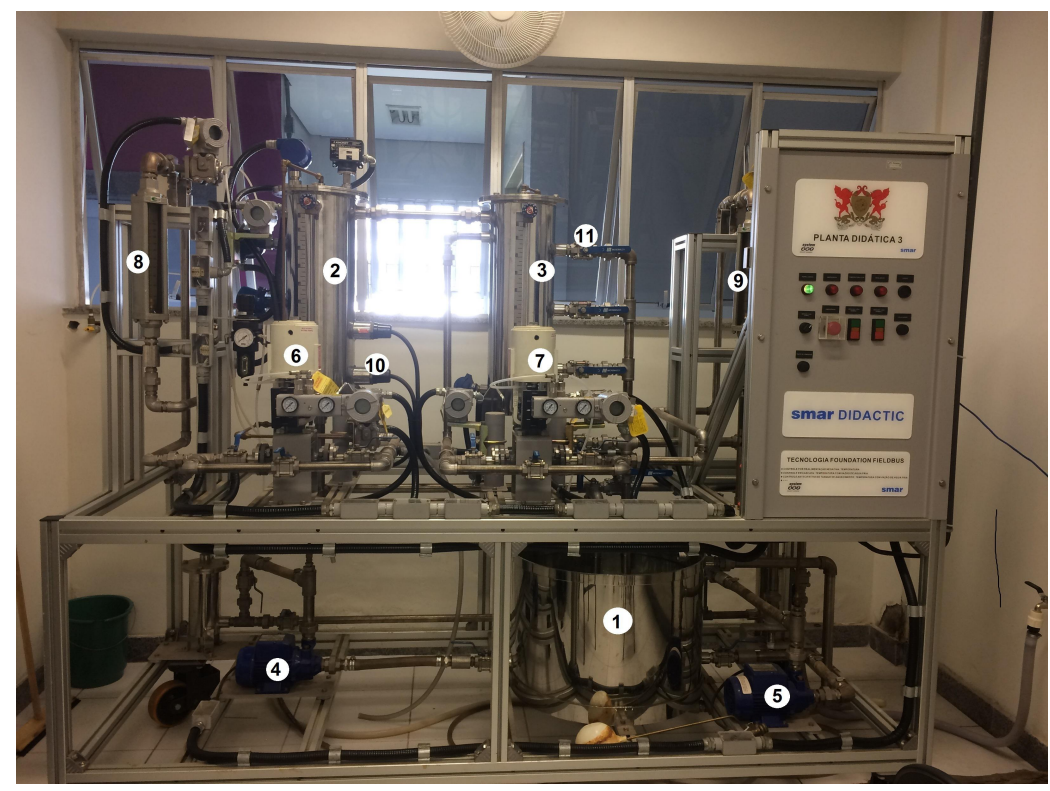

Figura 1 - Vista frontal da planta didática Smar PD3.

A Planta é monitorada e operada de uma estação, constituída de um microcomputador do tipo PC e um software de supervisão. Através do Sistema de Automação Industrial SYSTEM302, que utiliza a tecnologia Foundation Fieldbus, utiliza-se as ferramentas de configuração e operação para atuar nos registros modificando valores internos dos 
equipamentos e nos modos operacionais das malhas de controle (SMAR, 2004).

No sistema adotado, o tanque de mistura (3) recebe duas vazões de água, ambas provenientes inicialmente do tanque reservatório (1). A primeira vazão $\left(\mathrm{F}_{1}\right)$ é bombeada pela bomba (4) para o tanque de aquecimento (2), podendo ser regulada pela válvula (6) e observada pelo rotâmetro (8). No tanque de aquecimento (2), a água é aquecida pelas resistências (10) e segue para o tanque de mistura (3). A segunda vazão $\left(\mathrm{F}_{2}\right)$ é bombeada diretamente para o tanque de mistura (3) pela bomba (5) podendo ser regulada pela válvula (7) e observada pelo rotâmetro (9), similar ao de número (8). O tanque reservatório (1) recebe água diretamente da caixa d'água do departamento. Além disso, o tanque de mistura (3) possui capacidade total de 24 litros, não possui controlador de nível e a saída do fluxo ocorre por três válvulas manuais (11) posicionadas em diferentes alturas no tanque.

\subsection{Modelagem Experimental}

A modelagem experimental do processo de mistura no tanque (3) foi feita através de dois experimentos com aplicação de 3 degraus diferentes na vazão de água fria $\left(\mathrm{F}_{2}\right)$, sendo esses nos valores de 100, 200 e 400 L.h ${ }^{-1}$, em cada um dos experimentos. A saída do fluxo nesse tanque ocorreu pela válvula manual de posição intermediária localizada a $38 \mathrm{~cm}$ da base do tanque, mantendo-se um volume 2 igual a 13 litros no mesmo. O procedimento adotado em cada experimento apresentou as seguintes etapas:

1) Ajuste da vazão $\left(F_{1}\right)$ no tanque de aquecimento (2) para $200 \mathrm{~L} \cdot \mathrm{h}^{-1}$ e da temperatura $\left(\mathrm{T}_{1}\right)$ para set-point igual a $35^{\circ} \mathrm{C}$ pelo controlador PI implementado anteriormente na planta por Domingos et al. (2017);

2) Estabelecimento de um regime permanente de operação no tanque (2);

3) Ajuste da vazão $\left(\mathrm{F}_{2}\right)$ inicial igual a 200 L.h.h A temperatura $\left(\mathrm{T}_{2}\right)$ é aproximadamente igual a temperatura ambiente no dia do experimento;

4) Mistura das vazões $F_{1}$ e $F_{2}$ no tanque (3) e estabelecimento de um regime permanente de operação com uma temperatura de equilíbrio $\left(\mathrm{T}_{3}\right)$ aproximadamente igual a $31^{\circ} \mathrm{C}$;

5) Aplicação de um degrau de $100 \mathrm{~L} \cdot \mathrm{h}^{-1}$ na vazão $\mathrm{F}_{2}$ por aproximadamente 20 minutos até que o tanque (3) atinja novamente um regime permanente com uma nova temperatura de equilíbrio $\left(\mathrm{T}_{3}\right)$;

6) Ajuste da vazão $F_{2}$ de $300 ~ L . h^{-1}$ para a vazão inicial de 200L.h. ${ }^{-1}$. Em seguida, repetição das etapas 4 e 5 para os degraus de 200 e 400 L.h.' ${ }^{-1}$.

Ambos os tanques receberam agitação constante ao longo dos experimentos a fim de contribuir para a uniformidade da temperatura nos mesmos e para uma mistura perfeita no tanque (3). Os degraus escolhidos foram de acordo com a capacidade do tanque de mistura, a vazão total $\left(\mathrm{F}_{1}+\mathrm{F}_{2}\right)$ deve ser menor ou igual a $800 \mathrm{~L} \cdot \mathrm{h}^{-1}$ para que o volume 2 seja mantido no respectivo tanque. A Figura 2 apresenta a metodologia adotada. 


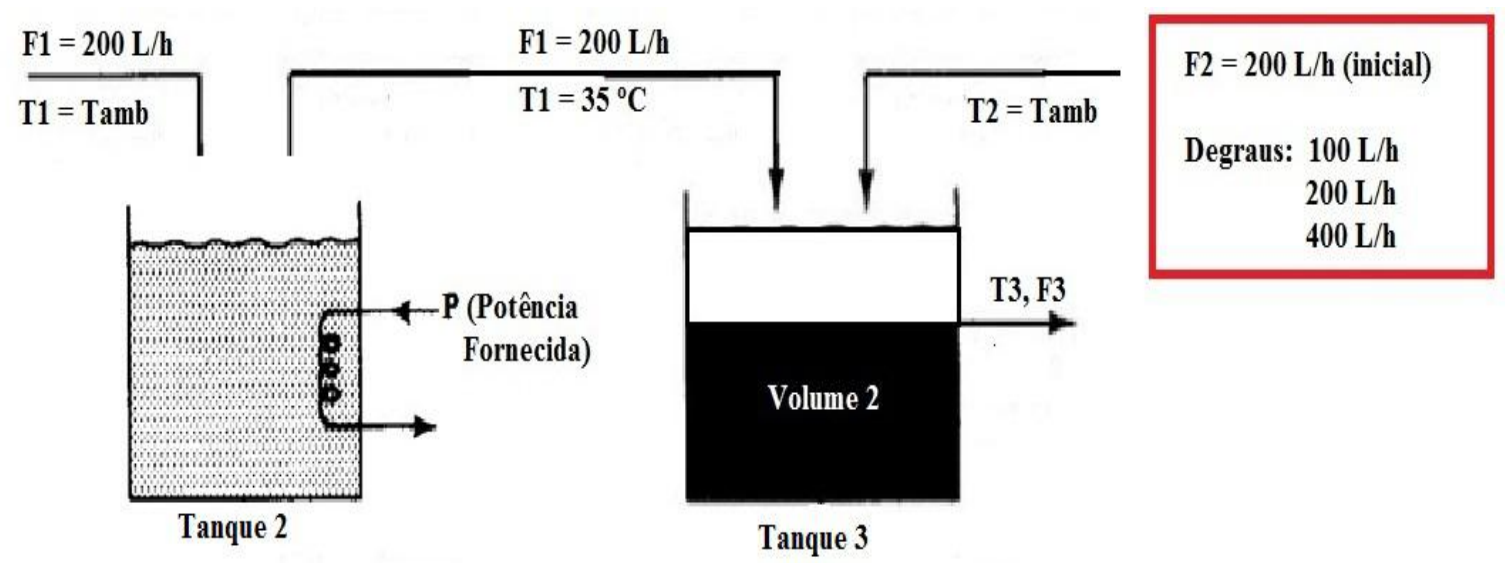

* Agitação constante nos dois tanques.

Figura 2 - Procedimento para modelagem experimental.

\section{RESULTADOS E DISCUSSÃO}

\subsection{Modelagem Teórica}

A modelagem teórica foi desenvolvida para ser utilizada durante a etapa de validação do modelo de precisão do processo, sendo comparada com os resultados obtidos da modelagem experimental e com os dados dos experimentos na planta. A modelagem abaixo foi baseada nos balanços de massa e energia para um tanque de mistura cilíndrico e com agitação constante, representado na Figura 3, podendo ser vista com mais detalhes em Stephanopoulos (1984, v. 1, p.64).

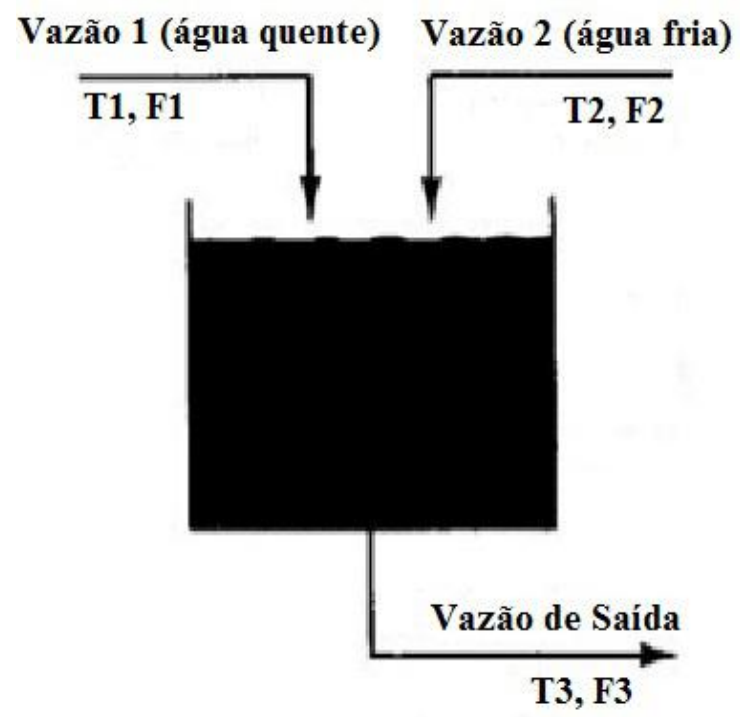

Figura 3 - Tanque de mistura. Fonte: Adaptado de Stephanopoulos (1984). 
O balanço de massa do processo pode ser descrito pela Equação 1:

$$
\frac{d(\rho V)}{d t}=\rho_{1} F_{1}+\rho_{2} F_{2}-\rho_{3} F_{3}
$$

Considerando que as densidades $\rho_{1}, \rho_{2}, \rho_{3}$ e $\rho$ são constantes e iguais, a Equação 1 pode ser simplificada para a seguinte:

$$
\frac{d(V)}{d t}=F_{1}+F_{2}-F_{3}
$$

Já o balanço de energia é representado pela Equação 3:

$$
\frac{d E}{d t}=\frac{d(U+K+P)}{d t}=\sum_{i=0}^{n} \rho_{i} F_{i} h_{i}-\sum_{j=0}^{m} \rho_{j} F_{j} h_{j} \pm q^{\prime \prime} \pm W_{s}
$$

Em que:

$\rho_{i}$ é a densidade do fluido de entrada $\mathrm{i}$;

$\rho_{j}$ é a densidade do fluido de saída $\mathrm{j}$;

$U$ é a energia interna do sistema;

$P$ é a energia potencial do sistema;

$K$ é a energia cinética do sistema;

$F_{i}$ é a vazão volumétrica do fluido de entrada i;

$F_{j}$ é a vazão volumétrica do fluido de saída j;

$h_{i}$ é a entalpia específica do material de entrada i;

$h_{j}$ é a entalpia específica do material de saída j; tempo;

$q^{\prime \prime}$ é a quantidade de calor trocado entre o sistema e as vizinhanças por unidade de

$W_{s}$ é o trabalho mecânico trocado entre o sistema e as vizinhanças por unidade de tempo.

Como o reservatório está parado, não recebe fonte de calor externa e não há troca de energia por trabalho com as vizinhanças, logo:

$$
\frac{d K}{d t}=\frac{d P}{d t}=q^{\prime \prime}=W_{s}=0
$$


Para sistemas líquidos, a energia interna pode ser aproximada com o valor da entalpia do reservatório:

$$
\begin{aligned}
& \frac{d U}{d t} \sim \frac{d H}{d t} \\
& H=\rho V C_{p}\left(T-T_{\text {ref }}\right)
\end{aligned}
$$

Onde:

$V$ é o volume do reservatório;

$C_{p}$ é a capacidade calorífica da substância;

$T$ é a temperatura do meio;

$T_{\text {ref }}$ é a temperatura de referência, que nesse caso será $0{ }^{\circ} \mathrm{C}$.

Considerando $C_{p}$ constante e igual para os fluidos de entrada e de saída, além disso, um sistema com mistura perfeita, na qual a temperatura do fluxo de saída é igual à do tanque. Substituindo a Equação 6 na Equação 3:

$$
\frac{d\left(\rho V C_{p} T_{3}\right)}{d t}=\rho F_{1} C_{p} T_{1}+\rho F_{2} C_{p} T_{2}-\rho F_{3} C_{p} T_{3}
$$

Dividindo tudo por $\rho C_{p}$ :

$$
\frac{d\left(V T_{3}\right)}{d t}=F_{1} T_{1}+F_{2} T_{2}-F_{3} T_{3}
$$

Pela regra da cadeia:

$$
\frac{d\left(V T_{3}\right)}{d t}=V \frac{d\left(T_{3}\right)}{d t}+T_{3} \frac{d(V)}{d t}=F_{1} T_{1}+F_{2} T_{2}-F_{3} T_{3}
$$

Substituindo a Equação 2 em 9 e rearranjando:

$$
V \frac{d\left(T_{3}\right)}{d t}=F_{1} T_{1}+F_{2} T_{2}-F_{1} T_{3}-F_{2} T_{3}
$$

Após linearização dos termos $F_{1} T_{1}, F_{2} T_{2}, F_{1} T_{3}$ e $F_{2} T_{3}$ pela Série de Taylor, obtém-se a Equação 11:

$$
\begin{aligned}
& \frac{V d\left(T_{3}\right)}{d t}=F_{1 s} T_{1}+\left(T_{1 s}-T_{3 s}\right) F_{1}+F_{2 s} T_{2}+\left(T_{2 s}-T_{3 s}\right) F_{2}-\left(F_{1 s}+F_{2 s}\right) T_{3}-T_{1 s} F_{1 s}-T_{2 s} F_{2 s}+ \\
& F_{1 s} T_{3 s}+F_{2 s} T_{3 s}
\end{aligned}
$$

As variáveis no estado permanente receberam o subíndices. 
Em regime permanente:

$$
\begin{aligned}
& \frac{V d\left(T_{3 s}\right)}{d t}=F_{1 s} T_{1 s}+\left(T_{1 s}-T_{3 s}\right) F_{1 s}+F_{2 s} T_{2 s}+\left(T_{2 s}-T_{3 s}\right) F_{2 s}-\left(F_{1 s}+F_{2 s}\right) T_{3 s}-T_{1 s} F_{1 s}- \\
& T_{2 s} F_{2 s}+F_{1 s} T_{3 s}+F_{2 s} T_{3 s}
\end{aligned}
$$

Sendo as variáveis desvio:

$$
\begin{aligned}
& T_{1}-T_{1 s}=T_{1}^{\prime} \\
& F_{1}-F_{1 s}=F_{1}^{\prime} \\
& T_{2}-T_{2 s}=T_{2}^{\prime} \\
& F_{2}-F_{2 s}=F_{2}^{\prime} \\
& T_{3}-T_{3 s}=T_{3}^{\prime}
\end{aligned}
$$

Subtraindo a Equação 11 da Equação 12 e simplificando através das Equações 13, 14 , 15,16 e 17 :

$$
\frac{V d\left(T_{3}^{\prime}\right)}{d t}=F_{1 s} T_{1}^{\prime}+\left(T_{1 s}-T_{3 s}\right) F_{1}^{\prime}+F_{2 s} T_{2}^{\prime}+\left(T_{2 s}-T_{3 s}\right) F_{2}^{\prime}-\left(F_{1 s}+F_{2 s}\right) T_{3}^{\prime}
$$

Definindo:

$$
\tau=\frac{V}{\left(F_{1 s}+F_{2 s}\right)} \quad K_{1}=\frac{F_{1 s}}{\left(F_{1 s}+F_{2 s}\right)} K_{2}=\frac{\left(T_{1 s}-T_{3 s}\right)}{\left(F_{1 s}+F_{2 s}\right)} \quad K_{3}=\frac{F_{2 s}}{\left(F_{1 s}+F_{2 s}\right)} K_{4}=\frac{\left(T_{2 s}-T_{3 s}\right)}{\left(F_{1 s}+F_{2 s}\right)}
$$

Aplicando a Transformada de Laplace na Equação 18 e rearrajando:

$$
T_{3}(s)=\frac{K_{1}}{\tau s+1} T_{1}(s)+\frac{K_{2}}{\tau s+1} F_{1}(s)+\frac{K_{3}}{\tau s+1} T_{2}(s)+\frac{K_{4}}{\tau s+1} F_{2}(s)
$$

Definindo as seguintes funções de transferência que descrevem a variação da temperatura no tanque de mistura:

$$
\begin{aligned}
& G_{1}(s)=\frac{T_{3}(s)}{T_{1}(s)}=\frac{K_{1}}{\tau s+1} \\
& G_{2}(s)=\frac{T_{3}(s)}{F_{1}(s)}=\frac{K_{2}}{\tau s+1} \\
& G_{3}(s)=\frac{T_{3}(s)}{T_{2}(s)}=\frac{K_{3}}{\tau s+1} \\
& G_{4}(s)=\frac{T_{3}(s)}{F_{2}(s)}=\frac{K_{4}}{\tau s+1}
\end{aligned}
$$


Logo,

$$
T_{3}(s)=G_{1}(s) T_{1}(s)+G_{2}(s) F_{1}(s)+G_{3}(s) T_{2}(s)+G_{4}(s) F_{2}(s)
$$

De acordo com Morais et al (2017), a temperatura no tanque de aquecimento $\left(\mathrm{T}_{1}\right)$ pode ser relacionada com a potência $(\mathrm{P})$ aplicada no tanque através da seguinte função de transferência:

$$
G_{0}(s)=\frac{T_{1}(s)}{P(s)}=\frac{K_{0}}{\tau_{0} s+1}
$$

Onde:

$$
\begin{aligned}
& K_{0}=\frac{1}{\rho C_{p} F_{1}} \\
& \tau_{0}=\frac{A h}{F_{1}}
\end{aligned}
$$

Sendo A a área da seção transversal do tanque e h a altura do tanque.

Seguindo as características físicas do tanque de aquecimento:

$$
\begin{aligned}
& A=3,42 \times 10^{-2} \mathrm{~m}^{2} \\
& h=0,7 \mathrm{~m} \\
& \rho=1000 \mathrm{~kg} \cdot \mathrm{m}^{-3} \\
& C_{p}=4186 \mathrm{~J} \cdot \mathrm{Kg}^{-1} \cdot{ }^{\circ} \mathrm{C}^{-1}
\end{aligned}
$$

E a vazão de entrada $\left(F_{1}\right)$ deve ser colocada $\mathrm{em} \mathrm{m}^{3} \cdot \mathrm{s}^{-1}$

Nesse caso, $\mathrm{T}_{3}$ pode ser relacionada com $\mathrm{P}$ através da associação em série entre as seguintes funções, como mostrado na Figura 4.

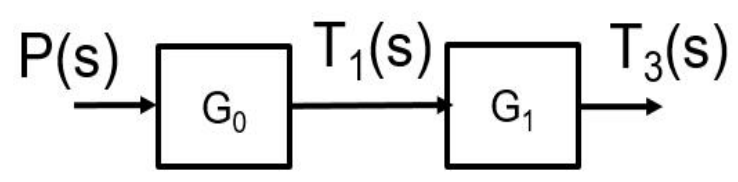

Figura 4 - Associação em série entre as funções de $T_{1}(s)$ e $T_{3}(s)$.

Logo:

$$
T_{3}(s)=G_{0}(s) G_{1}(s) P(s)
$$




$$
\frac{T_{3}(s)}{P(s)}=\frac{K_{0}}{\tau_{0} s+1} \frac{K}{\tau s+1}
$$

Por fim, obtemos a seguinte função de transferência para o processo de mistura no tanque (3):

$$
T_{3}(s)=G_{0}(s) G_{1}(s) P(s)+G_{2}(s) F_{1}(s)+G_{3}(s) T_{2}(s)+G_{4}(s) F_{2}(s)
$$

\subsection{Modelagem Experimental - Obtenção da Função de Transferência do Processo}

Com o procedimento descrito na seção 2.2 foi possível obter curvas da variação da temperatura $\left(\mathrm{T}_{3}\right)$ em resposta aos degraus aplicados na vazão $\left(\mathrm{F}_{2}\right)$. A partir disso, o ganho do processo e a constante de tempo foram calculados e a função de transferência de primeira ordem do processo foi determinada. A temperatura $\left(\mathrm{T}_{3}\right)$ era medida a cada segundo e os dados foram gravados em um arquivo no formato .csv gerado pelo programa da planta, ProcessView. $\mathrm{O}$ arquivo foi reorganizado para que as variáveis obtidas ficassem no formato variável desvio, para isso a temperatura inicial $\left(T_{3}\right)$ foi subtraída do valor de temperatura $\left(T_{3}\right)$ em cada instante. Por fim, os valores foram transferidos para o Matlab ${ }^{\circledR}$ e as curvas foram obtidas através da função Plot(y).

Para o Teste 1 , a temperatura inicial da vazão de água fria $\left(\mathrm{T}_{2}\right)$ foi igual a $25,3{ }^{\circ} \mathrm{C}$. A Figura 5 apresenta as curvas da temperatura $\left(T_{3}\right)$ no formato variável desvio em resposta aos degraus de 100, 200 e 400 L.h ${ }^{-1}$ aplicados na vazão $F_{2}$.

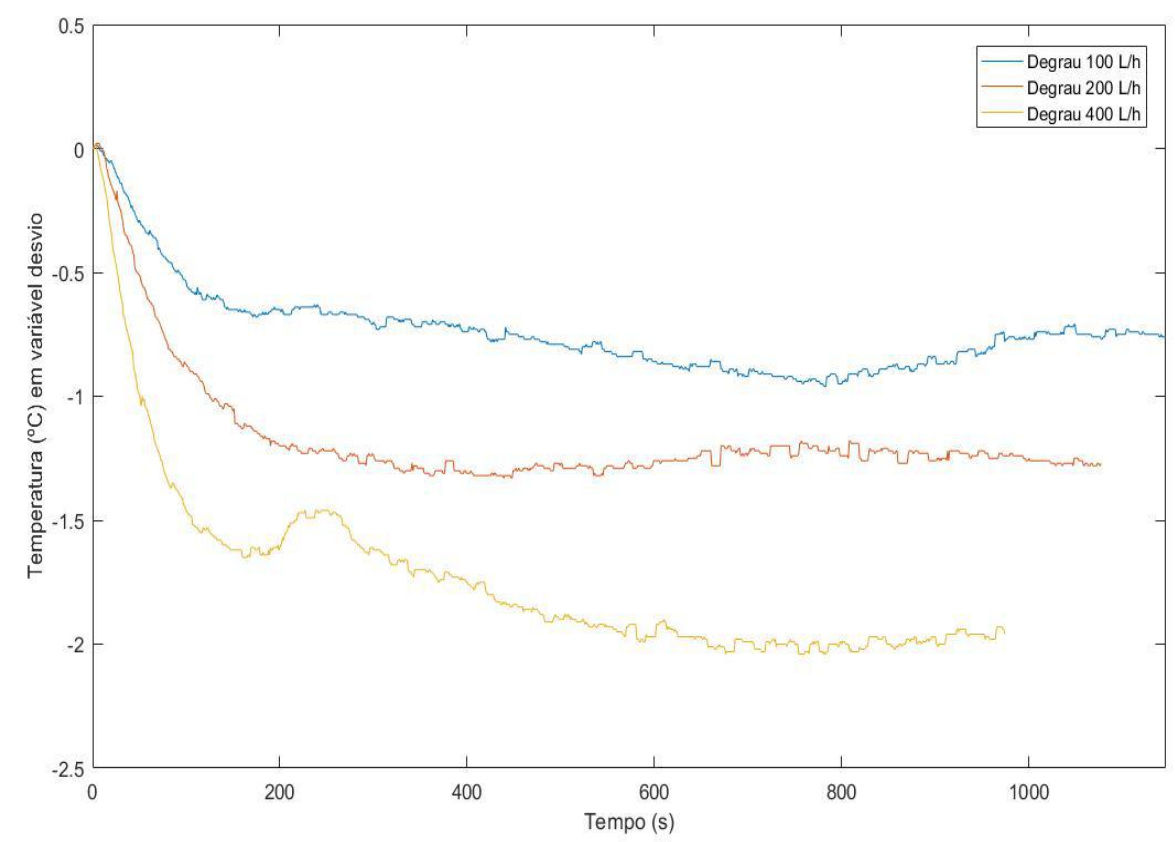

Figura 5 - Gráfico de temperatura $\left(\mathrm{T}_{3}\right)$ no formato variável desvio no tempo para os degraus de 100, 200 e 400 L.h ${ }^{-1}$ na vazão $F_{2}$, Teste 1. 
Como pode ser observado na Figura 5, para o degrau de 100 L.h ${ }^{-1}$ o valor da temperatura no regime permanente, em variável desvio, foi de aproximadamente $-0,67{ }^{\circ} \mathrm{C}$. Logo, o ganho do processo pode ser calculado pela Equação 31.

$$
K_{p 100}=\frac{\text { Temperatura no regime permanente }}{\text { degrau na vazão } F 2}=\frac{-0,67^{\circ} \mathrm{C}}{100 L \cdot h^{-1}}=-0,67 \times 10^{-2}{ }^{\circ} \mathrm{C} . h \cdot L^{-1}
$$

Para o cálculo da constante de tempo:

\section{$63,2 \%$ da temperatura no regime permanente $=0,632 \times\left(-0,67^{\circ} \mathrm{C}\right)=-0.42{ }^{\circ} \mathrm{C}$}

Como a constante de tempo é o valor de tempo para o qual essa variação de temperatura é alcançada, então, de acordo com o gráfico da Figura 5: $\tau_{1} \cong 73 \mathrm{~s}$. Similarmente, esses parâmetros foram determinados para as outras curvas. As funções de transferências (F.T) encontradas são apresentadas na Tabela 1.

Tabela 1 - Funções de transferência obtidas para cada degrau, Teste 1.

\begin{tabular}{cc} 
Degrau $\left(\mathbf{L} \cdot \mathbf{h}^{-1}\right)$ & F.T \\
\hline 100 & $\frac{-0,67 \times 10^{-2}}{73 s+1}$ \\
\hline 200 & $\frac{-0,6 \times 10^{-2}}{77 s+1}$ \\
& $\frac{-0,52 \times 10^{-2}}{78,5 s+1}$ \\
\hline 4
\end{tabular}

Já para o Teste 2 , a temperatura $\mathrm{T}_{2}$ inicial foi de $24,5^{\circ} \mathrm{C}$. A Figura 6 apresenta as curvas da temperatura $\left(\mathrm{T}_{3}\right)$ no formato variável desvio obtidas:

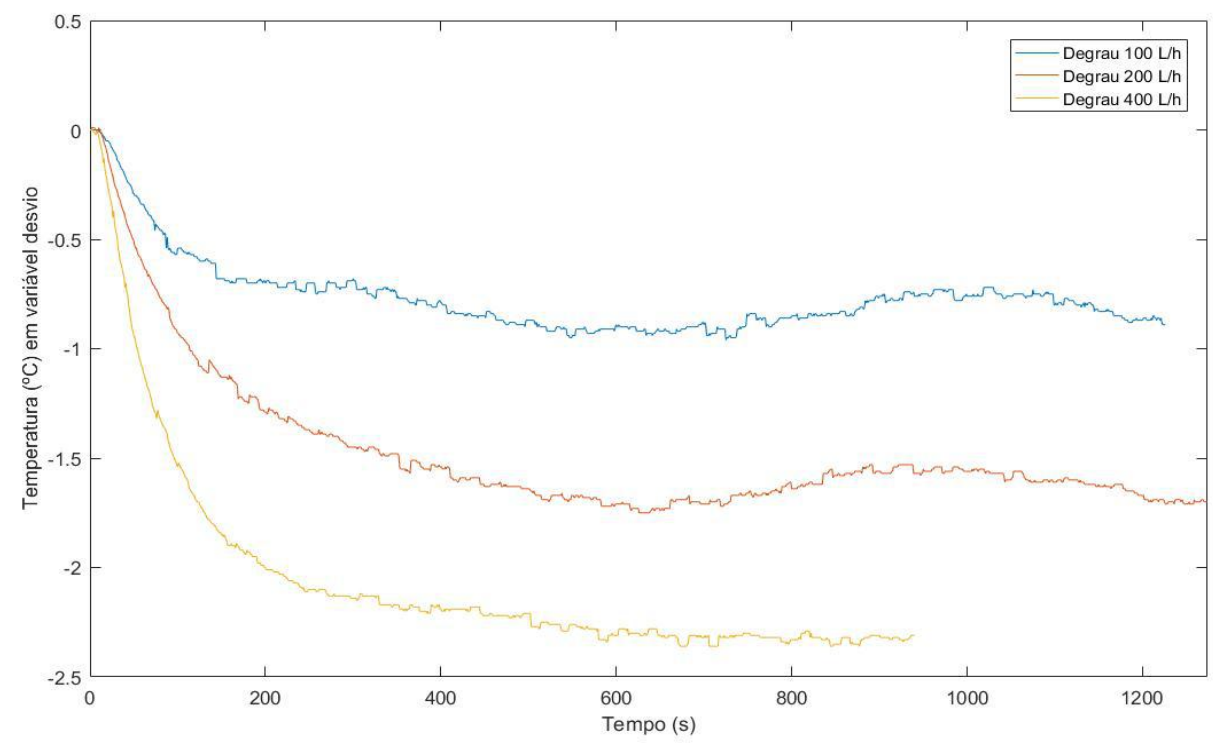

Figura 6 - Gráfico de temperatura $\left(\mathrm{T}_{3}\right)$ no formato variável desvio no tempo para os degraus de 100, 200 e 400 L.h ${ }^{-1}$ na vazão $F_{2}$, Teste 2. 
Nesse caso, a Tabela 2 apresenta as funções de transferência obtidas no Teste 2.

Tabela 2 - Funções de transferência obtidas para cada degrau, Teste 2.

\begin{tabular}{|c|c|}
\hline Degrau & F.T \\
\hline \multirow[t]{2}{*}{$100 \mathrm{~L} \cdot \mathrm{h}^{-1}$} & $-0,8 \times 10^{-2}$ \\
\hline & $\overline{88,31 s+1}$ \\
\hline \multirow[t]{2}{*}{200 L.h.' } & $-0,8 \times 10^{-2}$ \\
\hline & $114 s+1$ \\
\hline \multirow[t]{2}{*}{$400 \mathrm{~L} . h^{-1}$} & $-0,58 \times 10^{-2}$ \\
\hline & $91 s+1$ \\
\hline
\end{tabular}

De uma forma geral, os valores dos parâmetros encontrados no segundo teste foram um pouco maiores do que os do primeiro teste. Como demonstrado na modelagem teórica do sistema, considerando somente a função de transferência $G_{4}(s)$ que relaciona a vazão $\left(\mathrm{F}_{2}\right)$ com a temperatura $\left(\mathrm{T}_{3}\right)$.

$$
G_{4}(s)=\frac{T_{3}(s)}{F_{2}(s)}=\frac{K_{4}}{\tau s+1} \quad \text { sendo } K_{4}=\frac{\left(T_{2 s}-T_{3 s}\right)}{\left(F_{1 s}+F_{2 s}\right)} \text { e } \tau=\frac{V}{\left(F_{1 s}+F_{2 s}\right)}
$$

Além disso, para $\sum Q=0 \quad T_{3 s}=\frac{F_{1 s} T_{1 s}+F_{2 s} T_{2 s}}{\left(F_{1 s}+F_{2 s}\right)}$

O ganho do processo $\left(\mathrm{K}_{4}\right)$ é influenciado diretamente pela diferença entre a temperatura da água fria $\left(\mathrm{T}_{2 \mathrm{~s}}\right)$ e a temperatura $\left(\mathrm{T}_{3 \mathrm{~s}}\right)$ no regime permanente, esta última também é influenciada pela $\mathrm{T}_{2 \mathrm{~s}}$. Em módulo, quanto maior essa diferença, maior o ganho do processo. Como $\mathrm{T}_{1}$ é mantida constante e a diminuição de $\mathrm{T}_{2}$ contribui para o aumento da diferença $\left(\mathrm{T}_{2 \mathrm{~s}}-\mathrm{T}_{3 \mathrm{~s}}\right)$. Logo, sendo $\mathrm{T}_{2}$ no segundo teste cerca de um grau Celsius menor que no primeiro teste, era esperado que essa diferença fosse maior no segundo teste e o valor do seu ganho do processo consequentemente maior também.

Já em relação à constante de tempo, que é influenciada diretamente pelo volume no tanque (3), além de uma pequena diferença existente entre o volume em cada experimento ocasionada pela ausência de um controlador de nível no tanque, a diferença dos valores também pode ser associada com o nível de agitação perto do medidor de temperatura do tanque (3). O nível de agitação influenciou diretamente nas oscilações das temperaturas medidas ao longo de cada experimento pelo medidor e consequentemente na determinação do tempo para que o regime permanente fosse atingido na análise das curvas geradas. Essa agitação era feita através da injeção de ar na base do tanque, perto do medidor de temperatura, e o nível de agitação era regulado manualmente em cada experimento.

Observando a não linearidade do sistema com os valores dos parâmetros obtidos nas funções de transferência dos Testes 1 e 2, uma função de transferência (F.T) média entre os testes foi utilizada como aproximação para descrever o processo de mistura no tanque (3). A Tabela 3 apresenta a F.T média para cada experimento e a média para os dois experimentos. 
Tabela 3 - Funções de transferências médias.

\begin{tabular}{cc}
\hline Teste & F.T média \\
\hline 1 & $\frac{-0,59 \times 10^{-2}}{76,16 s+1}$ \\
\hline 2 & $\frac{-0,73 \times 10^{-2}}{99,77 s+1}$ \\
\hline Média & $\frac{-0,66 \times 10^{-2}}{88 s+1}$ \\
\hline
\end{tabular}

\subsection{Validação da Função de Transferência do Processo}

Para que a função de transferência média apresentada na Tabela 3 fosse validada, testes foram realizados com diferentes degraus em $\mathrm{F}_{2}$, dessa vez nos valores de $250 \mathrm{~L}^{\mathrm{h}} \mathrm{h}^{-1}$ e de 300 L.h ${ }^{-1}$. Novamente, os degraus escolhidos foram de acordo com a capacidade do tanque de mistura, a vazão total $\left(\mathrm{F}_{1}+\mathrm{F}_{2}\right)$ deve ser menor ou igual a $800 \mathrm{~L} . \mathrm{h}^{-1}$ para que o volume 2 seja mantido no respectivo tanque, além disso esses degraus são valores intermediários aos degraus de 100, 200 e 400 L.h ${ }^{-1}$ utilizados para a obtenção do modelo experimental. Em seguida, comparou-se os resultados desses experimentos com a função de transferência obtida pela modelagem teórica e com a função de transferência média obtida experimentalmente. As Figuras 7 e 8 apresentam os gráficos obtidos com a variável temperatura $\left(\mathrm{T}_{3}\right)$ no formato variável desvio, sendo a curva em azul a resposta para o experimento, em vermelho a resposta obtida pela função de transferência média e, em amarelo a resposta obtida pela função de transferência teórica para os degraus de 250 e 300 L.h ${ }^{-1}$ em $F_{2}$, respectivamente.

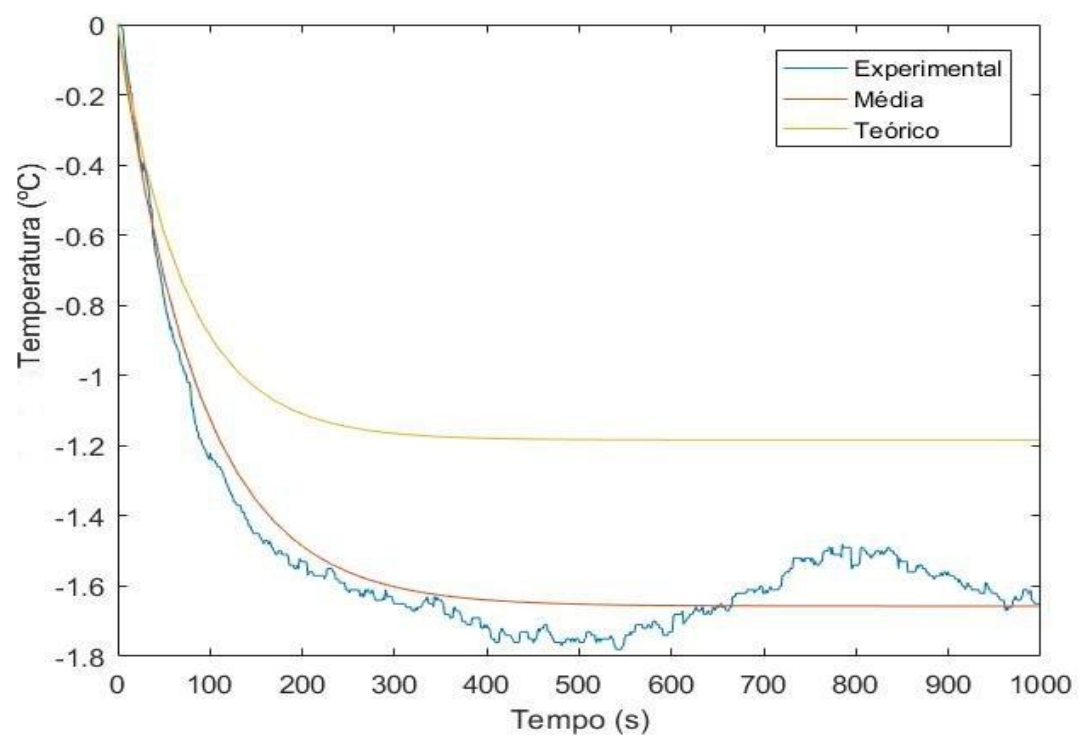

Figura 7 - Gráfico da validação para degrau de 250 L.h h $^{-1}$ em F $F_{2}$ 


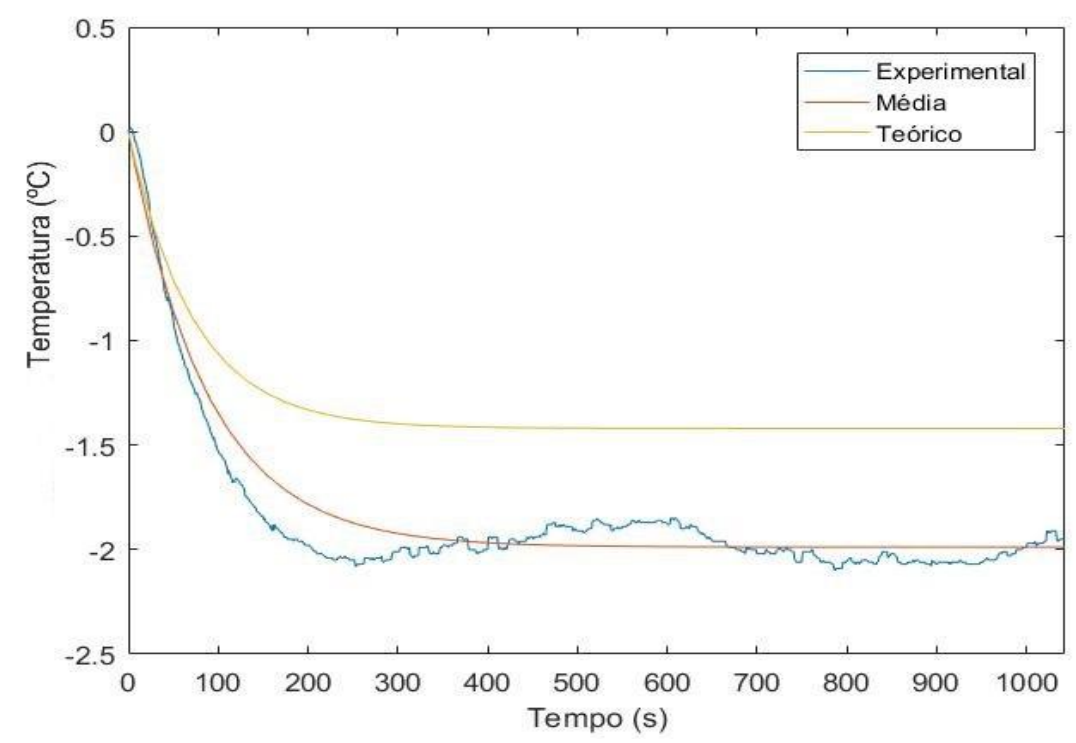

Figura 8 - Gráfico da validação para degrau de 300 L.h-1 em $\mathrm{F}_{2}$.

Como pode ser observado nas Figuras 7 e 8 , a resposta da modelagem teórica se distancia das respostas obtidas pela função de transferência média e nos experimentos. $\mathrm{O}$ ganho teórico é 38,2\% menor em relação à média nos dois experimentos. Comparando os experimentos com a média, na Figura 7 o ganho no experimento é $3,4 \%$ menor e na Figura 8 os ganhos são aproximadamente iguais.

A disparidade do ganho teórico em relação aos demais pode ser associada à diferença entre a $T_{2}$ considerada para o cálculo teórico da temperatura $T_{3}$ de equilíbrio na mistura, apresentado na Equação 32, e o valor real de $\mathrm{T}_{2}$ ao longo do experimento. Para o modelo teórico considerou-se uma $\mathrm{T}_{2}$ igual ao valor constante de $25^{\circ} \mathrm{C}$, entretanto nos testes de validação a temperatura $\mathrm{T}_{2}$ inicial era aproximadamente $24{ }^{\circ} \mathrm{C}$ e variava cerca de $2{ }^{\circ} \mathrm{C}$ em um período de 2 horas. A temperatura $\mathrm{T}_{2}$ depende das condições climáticas no dia de cada teste, já que a água fria é proveniente da caixa d'água que não é isolada termicamente e está exposta ao sol. Logo, o ganho real do processo também irá variar de acordo com as condições climáticas de cada dia.

Pelos resultados obtidos, o modelo teórico não é uma representação robusta do processo e não é aplicável a uma ampla faixa de operação da planta. A modelagem experimental consegue representar de uma forma mais adequada a faixa de operação da planta, portanto foi utilizada como modelo de precisão do processo para fazer a sintonia do controlador de temperatura no tanque de mistura.

\subsection{Obtenção da Função de Transferência de Malha Fechada}

A Figura 9 apresenta o diagrama de blocos genérico de um sistema de malha fechada com controle feedback. 


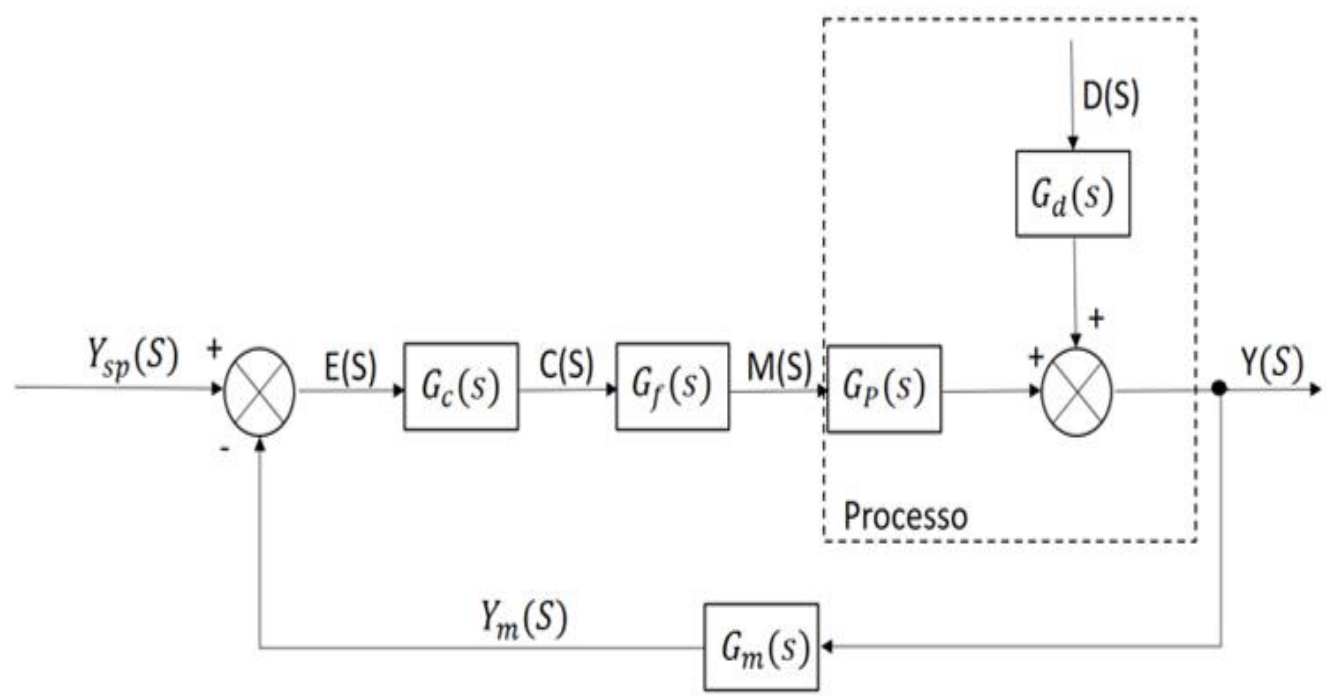

Figura 9 - Diagrama de blocos de um sistema de malha fechada.

Onde:

$Y(S)$ é a variável controlada;

$Y_{s p}(S)$ é o set-point;

$G_{p}(S)$ é a função de transferência do processo;

$G_{d}(S)$ é a função de transferência do distúrbio;

$M(S)$ é a variável de saída do elemento final de controle;

$D(S)$ é o distúrbio do sistema;

$Y_{m}(S)$ é a variável medida;

$G_{m}(S)$ é a função de transferência do dispositivo de medição;

$E(S)$ é o erro entre a variável medida e o set-point;

$C(S)$ é a variável de saída do controlador;

$G_{C}(S)$ é a função de transferência do controlador;

$G_{f}(S)$ é a função de transferência do elemento final de controle.

Mesmo a planta SMAR PD3 possuindo um controlador PID, optou-se por utilizar um controlador integral (PI) já que este é menos sensível a ruídos em relação ao controlador PID. Para o processo estudado tem-se que: 


$$
\begin{aligned}
& G_{p}(s)=\frac{K_{p}}{\tau_{p} s+1} \\
& G_{c}(s)=K_{c}\left(1+\frac{1}{\tau_{I} s}\right) \\
& G_{m}(s)=1 \\
& G_{f}(s)=1
\end{aligned}
$$

De acordo com Stephanopoulos (1984), a resposta genérica de malha fechada do processo pode ser descrita pela Equação 37:

$$
Y(s)=\frac{G_{p}(s) G_{f}(s) G_{c}(s)}{1+G_{p}(s) G_{f}(s) G_{c}(s) G_{m}(s)} Y_{s p}(s)+\frac{G_{d}(s)}{1+G_{p}(s) G_{f}(s) G_{c}(s) G_{m}(s)} D(s)
$$

Simplificando e rearranjando as Equações 33, 34, 35 e 36 na Equação 37:

$$
Y(s)=\frac{\tau_{I} s+1}{\left(\frac{\tau_{p} \tau_{I}}{K_{p} K_{c}}\right) s^{2}+\left(\frac{\tau_{l}}{K_{p} K_{c}}+\tau_{I}\right) s+1} Y_{s p}(s)+\frac{G_{d}}{\left(\frac{\tau_{p} \tau_{I}}{K_{p} K_{d}}\right) s^{2}+\left(\frac{\tau_{l}}{K_{p} K_{c}}+\tau_{I}\right) s+1} D(s)
$$

\subsection{Sintonia do Controlador PI}

O ajuste do controlador PI da planta foi realizado através do método do Lugar das Raízes, onde a alocação dos polos determina os valores de $\mathrm{K}_{\mathrm{C}}$ e de $\tau_{I}$ que possam tornar o sistema estável e com uma resposta rápida. Seguindo o método, as raízes da equação característica da função de transferência de malha fechada foram determinadas em função de um parâmetro do sistema, no caso o polo da função de transferência de malha aberta média apresentada na tabela 3 .

O polo de malha fechada escolhido inicialmente foi o polo dessa função, no caso $-\frac{1}{88}$ $\mathrm{rad} / \mathrm{s}$. Baseado na estratégia utilizada por Domingos et al. (2017) para implementação do controle de temperatura do tanque de aquecimento da planta, por estimativa o segundo polo foi escolhido com valor dez maior que o primeiro, sendo então igual a $-\frac{1}{8,8} \mathrm{rad} / \mathrm{s}$. Logo, o polinômio a seguir apresenta essas duas raízes:

$$
\left(s+\frac{1}{88}\right)\left(s+\frac{1}{8,8}\right)
$$

O polinômio 39 foi rearranjado e igualado à equação característica da função de transferência de malha fechada apresentada na Equação 38:

$$
774,4 s^{2}+774,4\left(\frac{1}{88}+\frac{1}{8,8}\right) s+1=\frac{\tau_{p}}{K_{p} K_{i}} s^{2}+\frac{\left(K_{p} K_{c}+1\right)}{K_{p} K_{i}} s+1
$$

Sendo: 
$\tau_{P}=88$

$K_{p}=-0,66 \times 10^{-2}$

Assim, isolando os termos na Equação 40, obtém-se:

$$
\begin{aligned}
& \frac{\tau_{P}}{K p K i}=774,4 \rightarrow K i=-17,15 \\
& \frac{(K p K c+1)}{K p K i}=774,4\left(\frac{1}{88}+\frac{1}{8,8}\right) \rightarrow K c=-1508,85 \\
& \tau_{I}=\frac{K_{c}}{K_{I}}=88
\end{aligned}
$$

Por fim, a função de transferência de malha fechada para o sistema com volume 2 no tanque de mistura (3) é dada pela Equação (44).

$$
Y(s)\left[V_{2}\right]=\frac{88 s+1}{774,4 s^{2}+96,8 s+1} Y_{s p}(s)+\frac{G_{d}}{774,4 s^{2}+96,8 s+1} D(s)
$$

Sendo a função de transferência de malha fechada de segunda ordem, os seguintes parâmetros podem ser determinados de acordo com a Equação 44:

$$
\tau=27,83_{\mathrm{s}} \text { e } \zeta=1,74
$$

Os valores encontrados para $\tau e \zeta$ caracterizam que uma resposta rápida $\mathrm{e}$ superamortecida será dada pelo controlador ajustado, já que $\tau$ possui um valor baixo e $\zeta>1$. Para comprovar isso, alguns testes foram realizados. Como a planta possui uma faixa de operação restrita, com vazões $F_{1}$ e $F_{2}$ de até 1000 L.h h $^{-1}$ cada uma, os set-points ajustados nos testes seguintes foram escolhidos de acordo com a temperatura $\left(\mathrm{T}_{2}\right)$ da vazão fria no dia de cada teste e a capacidade da planta e estimados através da Equação 32.

No primeiro teste, o controlador PI da planta SMAR foi ajustado com os valores de $\mathrm{K}_{\mathrm{C}}$ e de $\tau_{I}$ encontrados acima, assim como o set-point para a temperatura de mistura. A temperatura inicial $\left(\mathrm{T}_{2}\right)$ da vazão de água fria era $24,7^{\circ} \mathrm{C}$. A temperatura inicial da água no tanque de mistura era aproximadamente de $31{ }^{\circ} \mathrm{C}$ e o set-point foi ajustado para $28^{\circ} \mathrm{C}$. No tanque de aquecimento a temperatura foi mantida em $38{ }^{\circ} \mathrm{C}$ e sua vazão em aproximadamente $200 \mathrm{~L} . \mathrm{h}^{-1}$. A Figura 10 mostra o gráfico da temperatura da água no tanque de mistura pelo tempo. 


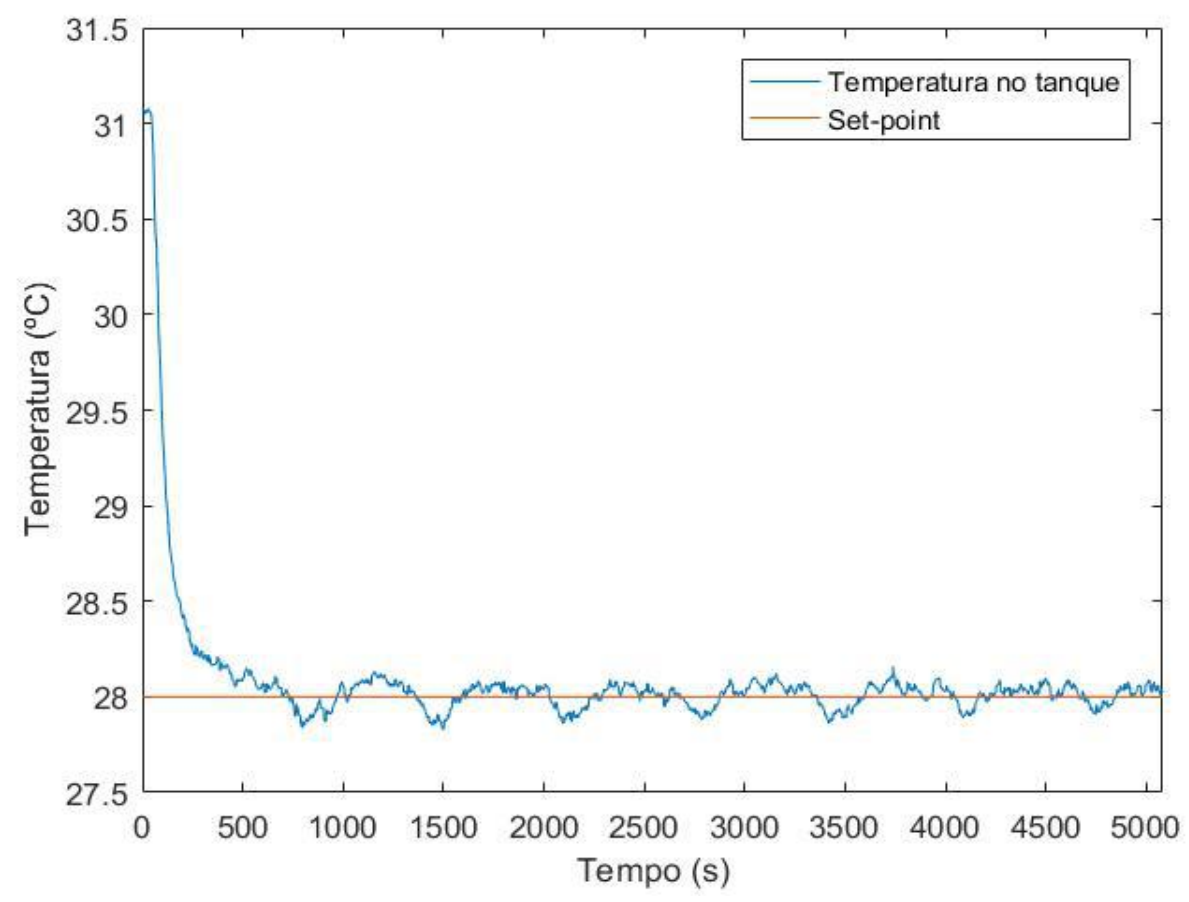

Figura 10 - Gráfico da resposta do sistema com o controle PI (Sintonia 1).

Como pode ser observado na Figura 10, a resposta é rápida, amortecida e estável. O valor do set-point é atingido em aproximadamente $10 \mathrm{~min}$ e a resposta não apresenta overshoot. Além disso, após atingir o regime permanente, a temperatura oscila em torno do set-point apenas $0,12{ }^{\circ} \mathrm{C}$ para cima e $0,15^{\circ} \mathrm{C}$ para baixo, variação que pode ser atribuída a distúrbios durante o experimento.

Com o intuito de aperfeiçoar o controlador para uma resposta um pouco mais rápida ainda, dois novos testes foram realizados com diferentes valores de $\mathrm{K}_{\mathrm{C}}$. A Figura 11 apresenta o segundo teste realizado, sintonia $2\left(K_{C}=-2250\right.$ e $\left.\tau_{I}=88\right)$. Nesse experimento, a temperatura inicial $\left(\mathrm{T}_{2}\right)$ da vazão de água fria era $24,6{ }^{\circ} \mathrm{C}$. A temperatura inicial da água no tanque de mistura era aproximadamente de $31{ }^{\circ} \mathrm{C}$ e o set-point foi ajustado para $28,5{ }^{\circ} \mathrm{C}$. No tanque de aquecimento a temperatura foi mantida em $38^{\circ} \mathrm{C}$ e sua vazão aproximadamente em 200 L.h $^{-1}$. 


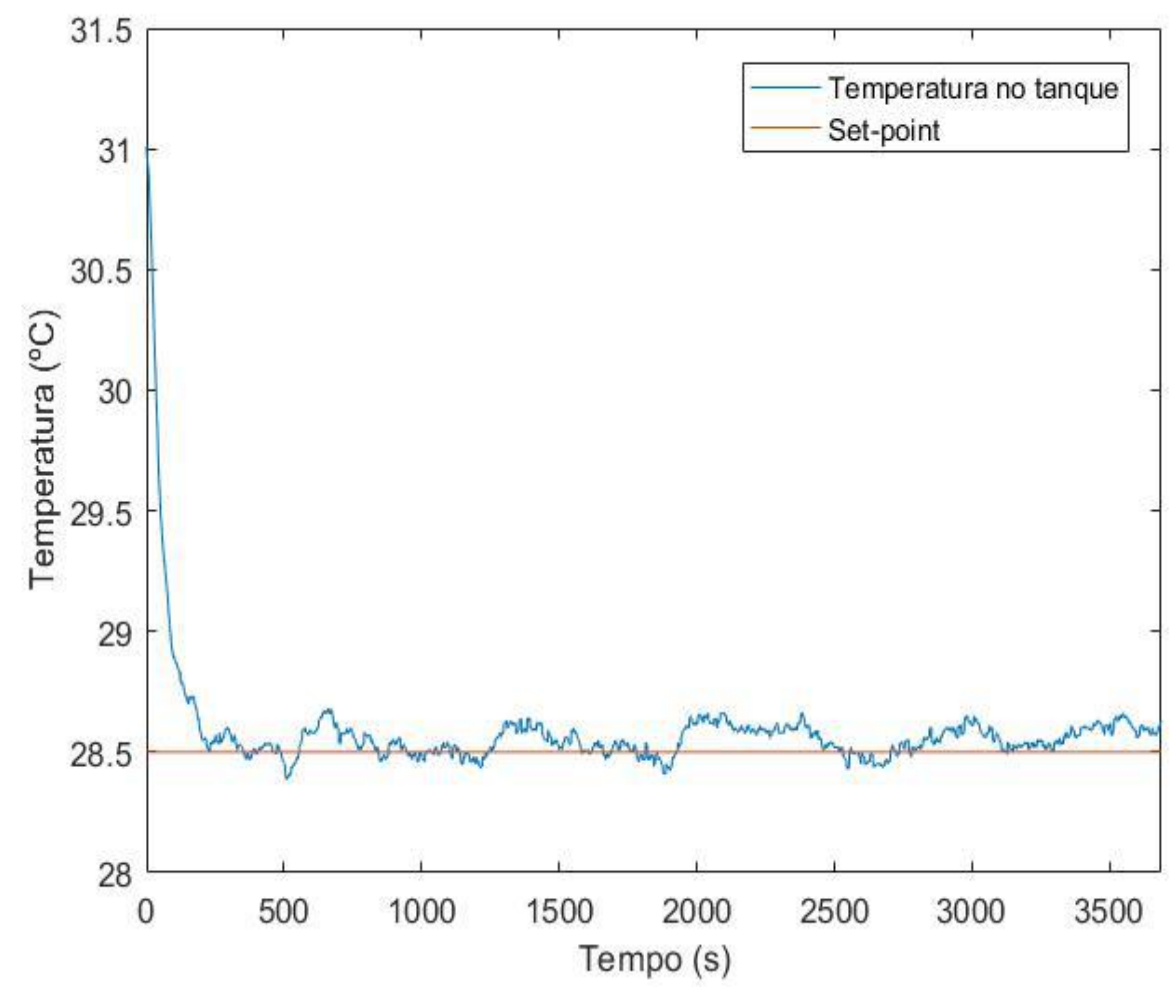

Figura 11 - Gráfico da resposta do sistema com o controle PI (Sintonia 2).

Como apresentado na Figura 11, o valor do set-point é atingido em aproximadamente 3,7 min, mais rápido do que na sintonia 1 . O sistema também continuou apresentando pequenas oscilações em torno do set-point, $0,18{ }^{\circ} \mathrm{C}$ para cima e $0,11{ }^{\circ} \mathrm{C}$ para baixo e não apresentou overshoot.

Uma terceira sintonia foi testada com $\mathrm{K}_{\mathrm{C}}=-1875$ e $\tau_{\mathrm{I}}=88$. Nesse experimento, a temperatura inicial $\left(\mathrm{T}_{2}\right)$ da vazão de água fria era $24,5{ }^{\circ} \mathrm{C}$. A temperatura inicial da água no tanque de mistura $\left(\mathrm{T}_{3}\right)$ era aproximadamente de $31{ }^{\circ} \mathrm{C}$ e o set-point foi ajustado para $28{ }^{\circ} \mathrm{C}$. Já no tanque de aquecimento, inicialmente a temperatura $\left(\mathrm{T}_{1}\right)$ foi mantida em $38{ }^{\circ} \mathrm{C}$ e sua vazão $\left(F_{1}\right)$ em aproximadamente $200 \mathrm{~L} \cdot \mathrm{h}^{-1}$, entretanto ao longo do teste algumas perturbações foram provocadas na vazão $F_{1}$ influenciando nas temperaturas $T_{1}$ e $T_{3}$. A Figura 12 apresenta o resultado. 

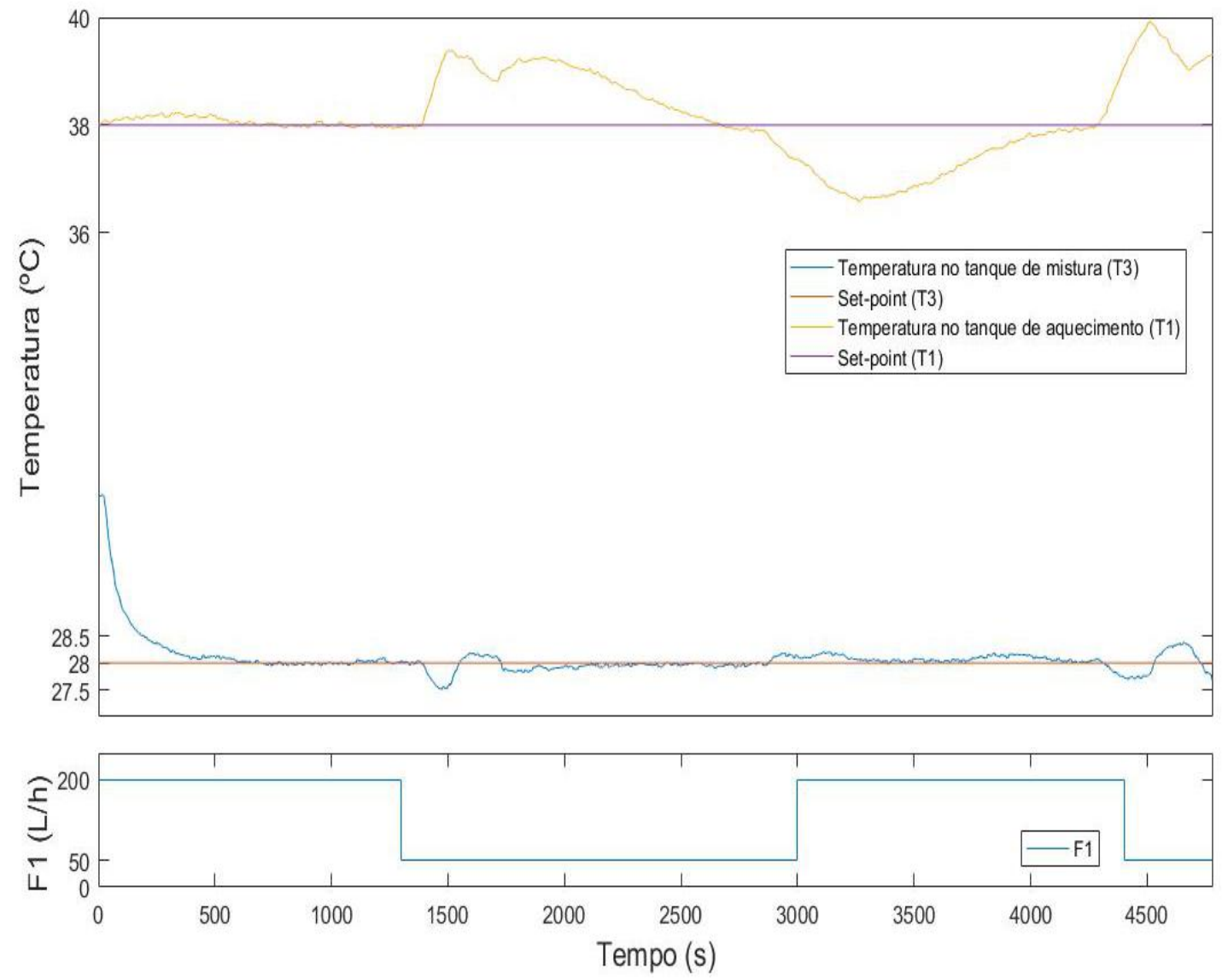

Figura 12 - Gráfico da resposta do sistema com o controle PI (Sintonia 3).

O tempo para atingir o regime permanente foi de aproximadamente $7 \mathrm{~min}$, como esperado é um valor intermediário entre os valores encontrados para as sintonias 1 e 2 já que a sintonia 3 apresenta um valor de $\mathrm{K}_{\mathrm{C}}$ intermediário entre as três sintonias. Já as oscilações em torno do set-point foram ainda menores do que nos outros dois testes, em torno de 0,02 ${ }^{\circ} \mathrm{C}$ para cima ou para baixo após os períodos com perturbações.

As perturbações ocorreram em 3 momentos durante o teste. A primeira delas foi no tempo de aproximadamente $1300 \mathrm{~s}$ quando a vazão $\mathrm{F}_{1}$ foi alterada bruscamente de $200 \mathrm{~L}^{-h^{-1}}$ para $50 \mathrm{~L} . \mathrm{h}^{-1}$. Nesse momento a temperatura no tanque de aquecimento $\left(\mathrm{T}_{1}\right)$ aumenta, com uma $F_{1}$ menor a temperatura no tanque de mistura $\left(T_{3}\right)$ diminui, entretanto o controlador rapidamente responde corrigindo $\mathrm{T}_{3}$ para o valor desejado de $28^{\circ} \mathrm{C}$. A segunda ocorre no tempo de aproximadamente $3000 \mathrm{~s}$ quando a vazão $\mathrm{F}_{2}$ foi ajustada novamente para o valor de $200 \mathrm{~L} . \mathrm{h}^{-1}$, nesse momento $\mathrm{T}_{1}$ diminui e $\mathrm{T}_{3}$ aumenta devido ao aumento de $\mathrm{F}_{1}$, mas novamente a temperatura retorna rapidamente para $28^{\circ} \mathrm{C}$. Uma última perturbação foi provocada no tempo de aproximadamente $4400 \mathrm{~s}$, alterando $\mathrm{F}_{1}$ para $50 \mathrm{~L} \cdot \mathrm{h}^{-1}$, entretanto isso ocorreu perto do final do teste e não foi possível concluir sobre a ação do controlador nesse momento.

Considerando essas perturbações, a resposta não apresentou overshoot e mesmo nos períodos em que a temperatura no tanque de aquecimento foi alterada por causa de variações da vazão $F_{1}$, o sistema respondeu de maneira rápida corrigindo para o valor desejado de 28 
${ }^{\circ} \mathrm{C}$ e com poucas oscilações, o que comprova uma alta estabilidade do controlador.

Para testar a validade das três sintonias para os outros dois possíveis volumes no tanque (3), os polos da Equação 38 foram calculados para o sistema com volume igual a 6,8 litros (volume 1) e com volume igual a 24 litros (volume 3). Para tal, os parâmetros $\left(\mathrm{K}_{\mathrm{C}}\right.$ e $\left.\mathrm{K}_{\mathrm{I}}\right)$ de cada sintonia do controlador foram mantidos e o valor de $\tau_{\mathrm{P}}$ para cada volume foi determinado. $\mathrm{O}$ ganho $\mathrm{K}_{\mathrm{p}}$ da função de transferência do processo não depende do volume e, portanto, foi considerado igual a $-0,0066^{\circ} \mathrm{C} . h . L^{-1}$ para ambos os volumes, este valor foi encontrado na modelagem feita com o volume igual a 13 litros (volume 2). A Tabela 4 apresenta os polos de malha fechada para cada volume, incluindo os do volume 2 usado na sintonização do controlador como forma de verificação da estabilidade.

Tabela 4 - Polos de malha fechada para cada volume e sintonia [rad/s].

\begin{tabular}{|c|c|c|c|}
\hline Volume & Sintonia 1 & Sintonia 2 & Sintonia 3 \\
\hline $\mathbf{1}$ & $-0,2259$ & $-0,3313$ & $-0,2779$ \\
$\left(\mathrm{~K}_{\mathrm{p}}=-0,0066^{\circ} \mathrm{C} . h . L^{-1}\right.$ & $-0,0108$ & $-0,0110$ & $-0,0109$ \\
$\left.\tau_{\mathrm{P}}=46 \mathrm{~s}\right)$ & & & \\
\hline $\mathbf{2}$ & $-0,1132$ & $-0,1687$ & $-0,1406$ \\
\hline$\left(\mathrm{K}_{\mathrm{p}}=-0,0066^{\circ} \mathrm{C} . h . L^{-1}\right.$ & $-0,0114$ & $-0,0114$ & $-0,0114$ \\
$\left.\tau_{\mathrm{P}}=88 \mathrm{~s}\right)$ & & & \\
\hline $\mathbf{3}$ & $-0,0549$ & $-0,0856$ & $-0,0701$ \\
$\left(\mathrm{~K}_{\mathrm{p}}=-0,0066^{\circ} \mathrm{C} \cdot h \cdot L^{-1}\right.$ & $-0,0127$ & $-0,0122$ & $-0,0124$ \\
\hline$\left.\tau_{\mathrm{P}}=162 \mathrm{~s}\right)$ & & & \\
\hline
\end{tabular}

Como pode ser observado na Tabela 4, os polos de malha fechada para os três volumes nas três sintonias foram reais e negativos, logo caracterizam que as três sintonias apresentam uma resposta de malha fechada estável para qualquer volume no tanque (3).

\section{CONCLUSÕES}

A modelagem experimental realizada foi adequada para descrever o processo de mistura no tanque (3), apresentando respostas aproximadamente iguais às respostas reais do sistema nos experimentos de validação da função de transferência do processo. O sistema de controle projetado se mostrou eficiente para controlar o processo de mistura, à medida que as respostas das sintonias testadas foram estáveis, rápidas e com poucas oscilações. Com isso, as três sintonias testadas podem ser adotadas na configuração do controlador PI da planta; especialmente a sintonia $2\left(\mathrm{~K}_{\mathrm{C}}=-2250\right.$ e $\left.\tau_{\mathrm{I}}=88\right)$ que atingiu o set-point desejado em menos de 4 minutos, para processos onde uma resposta mais rápida é desejada e a sintonia $3\left(\mathrm{~K}_{\mathrm{C}}=\right.$ 1875 e $\tau_{\mathrm{I}}=88$ ) para processos onde oscilações não são desejadas ou não são permitidas. 


\section{REFERÊNCIAS}

ANDRADE, A. B.; ARAÚJO, D. B. Desenvolvimento de um Simulador para a Planta Didática PD3 da SMAR Utilizando Estratégia de Controle Feedback e Cascata. 2013. 76 f. Monografia (Engenharia de Controle e Automação) - Instituto Federal de Educação, Ciência e Tecnologia Fluminense, Campos dos Goytacazes, RJ, 2013.

BERTACHI, A. H.; SILVA, L. R. B.; SUMAR, R. R.; ANGÉLICO, B. A.; GOEDTEL, A. Controle de um Processo Multivariável em uma Planta Didática Industrial Utilizando Redes Neurais. 2011. Universidade Tecnológica Federal do Paraná UTFPR, Cornélio Procópio, PR, 2011. Disponível em: < http://www.sbai2013.ufc.br/pdfs/5043.pdf >. Acesso em 04 Jul. 2017.

DOMINGOS, N; RODRIGUES, N. B.; NICACIO, J.; TÔRRES, A.. Planta Didática Smar PD3: Modelagem e Simulação do Tanque de Aquecimento - Parte B. The Journal of Engineering and Exact Sciences, Viçosa, MG, V. 3, N. 3, Mar. 2017. Disponível em: $<$ http://www.seer.ufv.br/seer/rbeq2/index.php/req2/article/view/161>. Acesso em: 17 Set. 2017.

GOMES, S. S. Desenvolvimento e Análise de Resultados entre os Controladores PID e Fussy para o Controle de Temperatura da Planta Piloto Didática SMAR (PD3) Utilizando a Estratégia Feedback. 2013. 50 f. Monografia (Engenharia de Controle e Automação) - Instituto Federal de Educação, Ciência e Tecnologia Fluminense, Campos dos Goytacazes, RJ, 2013.

KWONG, W. H. Introdução ao Controle de Processos e à instrumentação usando Scicos. Volume 1. São Carlos: EdUFScar, 2013. 147p.

OGATA, K. Modern Control Engineering. 5.ed. New Jersey: Prentice Hall, 2010.894p.

RODRIGUES DE MORAIS, C.; DOMINGOS, N; NICACIO, J.; TÔRRES, A.. Planta Didática Smar PD3: Modelagem e Simulação do Tanque de Aquecimento - Parte A. The Journal of Engineering and Exact Sciences, Viçosa, MG, V. 3, N. 3, Mar. 2017. Disponível em: <http://www.seer.ufv.br/seer/rbeq2/index.php/req2/article/view/167>. Acesso em: 17 Set. 2017.

SEBORG, D. A.; EDGAR, T. F.; MELliCHAMP, D. A. Process Dynamics and Control. 2. Ed. United States: John Wiley \& Sons, 2003.

SMAR. PD3 - Planta Didática HART, FOUNDATIONTM Fieldbus e PROFIBUS. 2004. Disponível em: $\quad<$ http://www.smar.com/brasil/produto/pd3-planta-didatica-hartfoundation-fieldbus-e-profibus $>$. Acesso em 10 Mar. 2017.

STEPHANOPOUlOS, G. Chemical Process Control: An Introduction to Theory and Practice. New Jersey: Prentice Hall, 1984. 696p. 